\title{
Dictynna
}

Dictynna

Revue de poétique latine

$14 \mid 2017$

Varia

\section{Heinze, Fraenkel e altre voci: contributi sparsi alla storia e al significato del dibattito su occasione e destinatario in Orazio lirico.}

\section{Mario Citroni}

\section{(2) OpenEdition \\ Journals \\ Edizione digitale \\ URL: http://journals.openedition.org/dictynna/1430 \\ DOI: 10.4000/dictynna.1430 \\ ISSN: $1765-3142$}

\section{Notizia bibliografica digitale}

Mario Citroni, « Heinze, Fraenkel e altre voci: contributi sparsi alla storia e al significato del dibattito su occasione e destinatario in Orazio lirico. », Dictynna [En ligne], 14 | 2017, mis en ligne le 01 décembre 2017, consulté le 10 décembre 2020. URL : http://journals.openedition.org/dictynna/1430 ; DOI : https://doi.org/10.4000/dictynna.1430

Questo documento è stato generato automaticamente il 10 décembre 2020.

\section{(c) $(1) \odot$}

Les contenus des la revue Dictynna sont mis à disposition selon les termes de la Licence Creative Commons Attribution - Pas d'Utilisation Commerciale - Pas de Modification 4.0 International. 


\title{
Heinze, Fraenkel e altre voci: contributi sparsi alla storia e al significato del dibattito su occasione e destinatario in Orazio lirico.
}

\author{
Mario Citroni
}

\section{NOTE DELL'AUTORE}

Sono lieto di dedicare queste riflessioni all'amico Alain Deremetz e alla sua inesausta passione per i problemi metodologici relativi all'analisi dei testi poetici.

1 1. Quasi all'inizio del suo Horace, e poi altre volte nel corso del volume, Eduard Fraenkel affermava il principio secondo cui l'interpretazione di una poesia di Orazio non può essere fatta dipendere da elementi esterni alla poesia stessa. Ogni singolo componimento deve essere interpretato soltanto sulla base di ciò che è contenuto nel suo testo, indipendentemente da ogni altra informazione desumibile dall'esterno di esso, e anche da altri carmi dello stesso Orazio. Fraenkel invitava il lettore che non condividesse questo principio a chiudere il suo libro e a non riaprirlo mai più. ${ }^{1}$

Questo categorico monito veniva a coincidere con un assioma fondamentale del New Criticism, la corrente critico-letteraria che si caratterizzava per la rivendicazione dell'autonomia del prodotto letterario dal contesto storico e (auto)biografico e dalla presunta intenzione autoriale, in nome di una esclusiva valorizzazione della sua elaborazione formale. In tale quadro critico, la situazione presupposta dal carme, la voce stessa dell'io lirico e del suo eventuale interlocutore sono considerati come elementi fittizi, come parte della costruzione artistica, e non si prestano dunque a essere integrati e sorretti da informazioni esterne, da referenti reali. Il New Criticism si era affermato negli Stati Uniti e poi in Inghilterra a partire dalla fine degli anni '30, e aveva dominato per alcuni decenni il panorama critico anglosassone. Gli studi di 
letteratura antica rimasero però a lungo estranei a questo movimento. Il volume di Steele Commager, The Odes of Horace, del 1962, fu salutato, al suo apparire, come prima testimonianza di un assai tardivo ingresso del New Criticism negli studi classici. ${ }^{2}$ In verità, per quanto riguarda il principio dell'autonomia del componimento lirico dai dati esterni Commager era molto più moderato di Fraenkel: fin dalla prefazione si dichiarava convinto che in molti casi il testo richiede, per essere compreso adeguatamente, la conoscenza di fatti noti ai contemporanei, e che noi dobbiamo riscoprire. Infatti altri due recensori che a proposito del libro di Commager si richiamano al New Criticism lo fanno proprio per segnalare la maggior cautela della posizione di Commager, e dunque la sua distanza rispetto ad esso, pur nel carattere innovativo del suo metodo di analisi formale della poesia oraziana. ${ }^{3} \mathrm{Negli}$ anni successivi le letture delle odi di Orazio, e in generale della poesia latina, improntate a un formalismo più o meno rigoroso, e $\mathrm{a}$ un più o meno rigoroso rifiuto di considerare, nell'interpretazione, dati e informazioni extra-testuali, si sono rapidamente moltiplicate, fondandosi sui diversi metodi critici di tipo formalista da allora succedutisi, e imponendosi a lungo come l'approccio predominante alla lirica e all'elegia latina.

3 L'Horace, finito di scrivere nel 1955, vedeva la luce nel 1957. Data l'autorevolezza di Fraenkel, e di quella sua grande opera, le sue drastiche ingiunzioni 'anticontestuali' hanno avuto a partire dagli anni ' 60 un ruolo non trascurabile nel legittimare presso il pubblico tradizionale degli studi di letteratura latina i nuovi approcci formalistici e anche nell'incentivarne lo sviluppo e l'applicazione alla poesia antica da parte di studiosi e critici. ${ }^{4}$ Di fatto, nel contesto di quelle letture formalistiche le parole di Fraenkel venivano spesso richiamate, e a loro volta acquistarono così molta notorietà. In realtà la posizione di Fraenkel non era influenzata significativamente dal New Criticism, o forse non ne era influenzata affatto. Comunque si fondava su presupposti teorici del tutto diversi.

4 In linea generale, è evidente che la metodica storicistica che Fraenkel praticava con fedeltà nei suoi studi, e nello stesso Horace, si collocava agli antipodi del New Criticism a cui quella presa di posizione sembrerebbe ascriverlo. Lo hanno già sottolineato sia Don Fowler, sia Joseph Farrell in due brevi interventi in proposito. ${ }^{5}$ Fowler e Farrell ritengono senz'altro che del New Criticism Fraenkel non avesse nozione, e Fowler precisa che quei concetti gli derivavano in realtà dalla tradizione romantica tedesca.

5 La prima di queste due affermazioni è credibile ma non è, a rigore, dimostrabile: è vero che, come abbiamo detto, il New Criticism non aveva una significativa presenza negli studi classici prima degli anni ' 60 . Ed è vero che Fraenkel non vi fa alcun cenno. Ma, essendo la corrente dominante nella critica letteraria anglosassone, non possiamo escludere che a Oxford egli ne avesse percepito alcune istanze, sia pure per via indiretta, ad esempio attraverso conversazioni con studiosi di letterature moderne. Ciò che conta è che, ad ogni modo, anche sullo specifico punto dell'autonomia del componimento poetico, la sua posizione se ne differenzia di fatto in modo sostanziale, come ora diremo.

6 Tra i molti recensori dell'Horace uno solo, se ho visto bene, richiamava il New Criticism, proprio per contrapporlo all'impianto fortemente tradizionale del metodo di analisi di Fraenkel. ${ }^{6}$ A.D. Leeman, in un breve intervento in cui tra l'altro criticava la presa di posizione di Fraenkel sull'autonomia del componimento poetico e rivendicava la rilevanza dello specifico contesto comunicativo contemporaneo per l'interpretazione 
del testo, osservava: "è chiaro che Fraenkel è stato influenzato da certe tendenze della moderna 'teoria della letteratura', che egli stesso però non menziona mai" - né Leeman si preoccupa di identificarle.

7 Sicuramente corretto è il riferimento alla tradizione dell'estetica romantica. I passi di W.A. Schlegel e di Coleridge che Fowler cita, pur pertinenti, hanno carattere in parte diverso, ${ }^{8}$ ma non sarebbe difficile trovare nei padri dell'estetica romantica e idealistica, $\mathrm{e}$ in primo luogo in Hegel, altre più esplicite e più generali proclamazioni della autonomia del discorso lirico dai dati concreti di esperienza, $i$ quali hanno in esso rilievo solo in quanto siano stati i generatori della esperienza soggettiva che trova espressione nel carme, e che devono essere interamente assorbiti in quell'esperienza, e nel testo del carme. ${ }^{9}$

Ma è Fraenkel stesso a offrirci una traccia che ci riconduce appunto alla critica romantica e non alla "moderna teoria della letteratura" cui pensava Leeman. Fraenkel, infatti cita, con molti elogi, come predecessore nell'affermazione del principio dell'autonomia del componimento lirico dai dati contestuali, uno studio oraziano di Philipp Buttmann (1764-1829), filologo e linguista, allievo di Heyne, autorevole membro dell'ambiente intellettuale berlinese tra fine '700 e inizi ' 800 , partecipe della Berliner Aufklärung e legato a personaggi quali Wolf, von Humboldt, Schleiermacher, Savigny. È l'ambiente in cui maturava parte importante della grande filologia tedesca, e in cui la potenza dell'erudizione era animata dagli ideali romantici dell'antico e da un'estetica di tipo idealistico. Nello studio citato da Fraenkel, Buttmann pronuncia varie affermazioni sul carattere meramente "ideale" degli oggetti e delle persone cui si riferisce la lirica oraziana, che andrebbero intesi non come dati storici ma come libere creazioni dal poeta, alla stregua della sua libera creazione e scelta delle immagini e delle parole. Perciò la comprensione della bellezza di un'ode di Orazio non potrebbe dipendere da un dato storico ad essa esterno. ${ }^{10}$

9 Eppure anche questo accostamento alla poetica idealistica e romantica cui, attraverso il richiamo a Buttmann, ci indirizza lo stesso Fraenkel, rischia di essere parzialmente fuorviante. Nelle analisi di Fraenkel, oggetti e persone cui i testi oraziani si riferiscono sono per lo più analizzati come dati storici e (auto)biografici, non come creazioni meramente ideali e fantastiche. Fraenkel non fondava la sua rivendicazione della autonomia del carme da elementi ad esso esterni sul principio estetico generale della autonomia creatrice del poeta, bensì sulla considerazione di quella che egli considerava una intenzione che definirei 'strategica' e 'pragmatica' del poeta: è l'intenzione di rendere il proprio discorso fruibile per un pubblico universale a indurre l'autore a rendere il discorso stesso sistematicamente autonomo da riferimenti che ne ostacolerebbero la leggibilità nei suoi aspetti rilevanti e sostanziali. Questa intenzione, e questa prassi, dell'autore a sua volta, per Fraenkel, ha un presupposto macrocontestuale che è di ordine propriamente storico: il passaggio dalla fase orale alla fase letteraria quando, a partire dalla Grecia del V secolo, "l'emancipazione della poesia dalle condizioni di vita di una precisa società era compiuta" e il poeta non avrebbe più scritto per un pubblico locale e limitato, ma per un pubblico universale, presente e futuro, delle più diverse regioni del mondo. ${ }^{11}$ Un pubblico, a quel punto, di lettori di libri e non più di uditori di recitazioni e di canti facenti parte integrante di momenti topici della vita della comunità quali simposii, riti religiosi e civili, adunanze civiche ecc. Il poeta, nel riutilizzare le forme tradizionali costituitesi per quelle situazioni specifiche di una particolare comunità in carmi ora destinati a un pubblico universale, 
cioè al pubblico del libro, sa di dover introdurre nel carme, ai fini della sua piena comprensione da parte di tale pubblico, quegli elementi contestuali necessari alla sua comprensione che nella fase orale erano nella disponibilità del pubblico locale, partecipe degli eventi in cui il carme era generato e comunicato.

10 L'idea della autonomia del carme oraziano derivava dunque a Fraenkel in primo luogo dalla consapevolezza del grande mutamento intervenuto con il passaggio dalla comunicazione orale a quella libraria: una consapevolezza che era maturata da tempo nella tradizione degli studi filologici classici: Fraenkel stesso rinviava, in proposito, a scritti già allora 'classici' di K.O. Müller, Reitzenstein, Wilamowitz collocati tra la metà dell'Ottocento e i primi anni del Novecento. ${ }^{12}$ Questa consapevolezza era un risultato dell'approccio genuinamente storicistico degli studi filologici in quella generazione, e si congiungeva con il convincimento romantico della autenticità nativa della letteratura greca e della cultura greca di contro al carattere derivato e libresco della letteratura e della cultura romana. Un'idea tuttora operante, anche a seguito del vasto sviluppo degli studi di oralistica che hanno esaltato la connessione della lirica greca arcaica con i riti della comunità, a fronte di una letteratura romana che di questa connessione vitale sarebbe priva. ${ }^{13}$ Va però anche segnalata la tesi in certo senso opposta di Paul Allen Miller che, basandosi sulla stessa contrapposizione, vede nel carattere librario della poesia romana, distaccata dalla ritualità, la condizione che, in positivo, avrebbe consentito la nascita, con Catullo, di una poesia introspettiva per la prima volta veramente corrispondente all'idea moderna di lirica, in quanto fondata sua costruzione di un "io" poetico le cui problematiche e le cui contraddizioni si seguono nel corso dello sviluppo di una raccolta poetica. ${ }^{14}$

11 Fraenkel, che pure è un grande ammiratore di Orazio e della cultura romana, individua anch'egli continuamente nella poesia oraziana una sfasatura tra le forme letterarie greche, desunte da una cultura in cui erano motivate da un contesto sociale e religioso preciso di cui erano parte integrante, e il loro reimpiego letterario e libresco nella poesia ellenistica e romana. Egli crede peraltro di riconoscere nel percorso poetico di Orazio una graduale maturazione verso una poesia personale-introspettiva e verso l'espressione di una genuina, commossa emozione per la grandezza della Roma augustea. Caratteristica è la sua interpretazione del Carme Secolare, che egli considera un capolavoro della più autentica poesia di Orazio, e cerca al tempo stesso di negare che fosse funzionalizzato direttamente al rito, benché in questo caso tale funzionalizzazione sia esplicitamente documentata dalle fonti storiche ed epigrafiche. ${ }^{15}$

12 Anche per il 'romantico' Buttmann i poeti greci rappresentavano persone e cose vere in quanto erano "poeti della naturalezza", mentre in Orazio "argomenti e persone appartengono all'arte", sono frutto di invenzione e emancipati dalla dimensione della realtà storica. Fraenkel invece ammette che, almeno in un certo numero di casi, e tipicamente nel caso delle Epistole, il componimento oraziano, come quello catulliano, va considerato come un Gelegenheitsgedicht, in quanto prende spunto da un'occasione reale. Ma, per Fraenkel, nel momento in cui il poeta lo destina, consapevolmente, a una fruizione libraria, e dunque universale, deve conferire al carme occasionale, conformemente all'idea goethiana di Gelegenheitsgedicht, un significato universale che trascende l'occasione e la cui comprensione non deve dipendere da elementi fattuali esterni al testo stesso (p. 313 s.). Il carme deve appunto essere, secondo la formula continuamente ribadita da Fraenkel, "self-contained". Tanta attenzione a questa specifica conseguenza del passaggio alla comunicazione libraria, e tanta attenzione al 
pressoché sconosciuto saggio di Buttmann in cui era affermato lo stesso principio sia pur su basi in buona parte diverse, si spiegherebbero meglio ammettendo che Fraenkel potesse aver colto nell'atmosfera culturale del suo tempo quella nuova sensibilità per l'autonomia del testo che il New Criticism aveva potentemente diffuso.

13 Oggi, dopo la grande fioritura di letture oraziane e catulliane di tipo formalistico che seguì, e che continua in parte ancora, e dopo il successivo tramonto del loro predominio di fronte a un diffuso recupero dell'attenzione ai contesti storici e sociali della produzione letteraria, questo episodio iniziale ci appare molto lontano. Ma permane, credo, un suo interesse per la storia dei nostri studi, perché, come ho detto, la convergenza allora verificatasi tra il filologo tradizionale, empirico e storicista, e gli incipienti formalismi, ha avuto la sua parte nel legittimare anche le letture più rigidamente formalistiche presso gli ambiti accademici dei classicistici, e viceversa nel delegittimare gli approcci critici, quale quello da me stesso assunto fin dalla fine degli anni ' 70 , che intendevano recuperare una rilevanza interpretativa alle originarie situazioni occasionali delle liriche oraziane e al ruolo dei destinatari. Inoltre, benché quel dibattito, espresso in quei termini, appaia oggi remoto, la sostanza del problema rimane aperta, e la posizione assunta da Fraenkel continua a vivere in quanto collocata, in grande evidenza, in un libro che è presenza stabile nelle mani di tutti gli studiosi di Orazio.

14 Al fondo, il problema del rapporto tra discorso lirico e occasione, specialmente se nell'occasione vengano coinvolti personaggi reali, è parte del più ampio problema del rapporto tra discorso poetico e dati di realtà: un grande problema generale di estetica, e di filosofia (rapporto tra realtà e rappresentazione) che non può avere soluzione semplice e che di fatto continua a essere l'oggetto di una intensa riflessione teorica. ${ }^{16}$ Per quanto riguarda la situazione della lirica antica, il dibattito continua a coinvolgere, o a presupporre, le opposizioni oralità vs. scrittura, Grecia vs. Roma, lirica greca vs. lirica moderna:, per lo più interpretate in senso esclusivo. Solo in tempi recenti qualche voce ha cercato di mettere in discussione la validità storica ed ermeneutica di queste opposizioni, se intese con rigidità. ${ }^{17}$ Nelle pagine che seguono intendo a mia volta contribuire a una visione più sfumata di esse.

15 2. L'opposizione tra il pubblico locale, particolare, che si presume direttamente coinvolto nel concepimento stesso del carme oltre che nella sua performance nella fase orale e il pubblico universale del libro era alla base anche del celebre saggio di Richard Heinze, Die horazische Ode, del 1923, che costituisce ancora un punto di riferimento nel dibattito sul ruolo dell'occasione nella lirica oraziana. ${ }^{18}$ Impostazione e linea argomentativa di Heinze erano però del tutto proprie e originali, anche in quanto improntate a un sistematico formalismo, insueto per il suo tempo, che ha poi favorito la fortuna del suo contributo presso gli interpreti recenti di Orazio. Viceversa, proprio l'impianto formalistico dell'argomentazione probabilmente spiega perché lo storicista Fraenkel, nel suo Horace, non faccia riferimento al saggio di Heinze, ove pure si escludeva che la comprensione di un'ode potesse dipendere da dati occasionali non presenti nel testo. Ma, a differenza che per Fraenkel, per Heinze questa considerazione discendeva dall'idea che ogni occasione presupposta dal carme oraziano dovesse essere considerata come fittizia, come parte delle convenzioni formali adottate dal poeta per le odi.

16 Heinze muoveva da un'opposizione tra lirica oraziana e lirica moderna sulla base del carattere sistematicamente allocutivo della lirica oraziana a fronte del carattere, a suo 
dire, pressoché sistematicamente monologico della lirica moderna. Lirica moderna, in senso proprio, per Heinze significava, 'romanticamente', lirica introspettiva: un modello di lirica che non gli appariva compatibile con la forma allocutiva, che coinvolge, strutturalmente, un interlocutore. La diversità più marcata rispetto alla lirica moderna consiste, secondo Heinze, nel fatto che Orazio non introduce l'interlocutore per valersene come figura di un mero ricettore dell'espressione della propria interiorità, bensì per condizionarne la volontà $o$, più raramente, per coinvolgerlo in una propria espressione di volontà. Questa caratteristica veniva spiegata da Heinze come una eredità della lirica greca che, in quanto eseguita oralmente in situazioni determinante davanti a un uditorio, svolgeva appunto una reale funzione di raccomandazione, esortazione, consolazione, ammonizione nei confronti di esso. Secondo Heinze, venuta meno la dimensione orale, la modalità allocutiva era divenuta anacronistica: una finzione ormai priva di referenti sostanziali. Ogni ode oraziana simula, per Heinze, di essere cantata con l'accompagnamento di uno strumento musicale, davanti all'interlocutore per condizionarne la volontà in una situazione occasionale determinata. Ma canto, accompagnamento musicale, presenza dell'interlocutore, intenzione di condizionarne la volontà e occasione in cui l'ode è eseguita sono finzioni, adottate dal poeta per conformarsi alle modalità proprie dei suoi dei suoi modelli greci, per i quali erano invece concreta realtà. È dunque una finzione che "chi ascolta debba riconoscere dai canti la situazione in cui essi si sono generati" (p. 188). Orazio, per Heinze, pretendeva troppo dalla fantasia dei suoi lettori, che per comprendere le sue odi avrebbero dovuto immergersi negli usi, per loro esotici, dei lirici greci. Di qui lo scarso successo incontrato da Orazio lirico presso il pubblico contemporaneo.

17 La tesi di Heinze è stata per lo più accolta, almeno nei suoi tratti essenziali, dalla critica successiva, ${ }^{19}$ ed è stata per lo più accolta anche la più ampia idea di un'opposizione tra lirica antica, nella sua fase orale, in cui l'allocuzione aveva funzione reale, e lirica moderna, in cui l'allocuzione, quando c'è, è un relitto formale derivato dalla tradizione classica. In Heinze, la lirica di Orazio, in quanto prodotta e fruita come testo librario, fa già parte della lirica moderna, in cui l'allocuzione è eredità formale priva di funzione reale, ma si oppone alla lirica moderna in quanto mantiene la modalità allocutiva in modo sistematico: una caratteristica formale che ne intralcia quello sviluppo monologico che solo sarebbe proprio della vera lirica.

Queste contrapposizioni devono essere sfumate. In primo luogo, è discutibile che la modalità allocutiva sia estranea alla lirica moderna. Dipende da cosa si intende per 'moderno'. Dal XV al XVIII secolo la lirica è stata comunemente allocutiva, anche perché, come la lirica antica, è stata spessissimo occasionale. Il romanticismo pronunciò la condanna della poesia occasionale: una condanna cui Goethe reagì rivalutando provocatoriamente l'occasione come condizione addirittura necessaria della poesia, ma a sua volta a condizione che l'occasione, secondo un principio a sua volta genuinamente romantico, fosse totalmente riassorbita nella soggettività e unicità dell'esperienza che essa aveva suscitata nel poeta. Prima della svolta romantica era invece del tutto normale che una lirica si collocasse in un contesto comunicativo occasionale. Sostenere, come fa Heinze, che, nella misura in cui una lirica si rivela dipendente da una situazione contestuale occasionale, essa non è più 'lirica' in senso proprio, significa presupporre che lirica in senso proprio sia solo la lirica in senso romantico e post-romantico: significa cioè mettere in atto un arrischiato anacronismo 
critico. Senza dire che esempi di poesia romantica, post-romantica e contemporanea atteggiata in forma allocutiva sono in realtà frequentissimi. ${ }^{20}$

Una forte valorizzazione del ruolo dell'apostrofe, e dunque del ruolo della seconda persona, nella lirica moderna, romantica e post-romantica è stata proposta da Jonathan Culler in un saggio molto noto, originariamente pubblicato nel $1977,{ }^{21}$ poi riedito, e ora largamente rielaborato nel suo recente volume di teoria generale della lirica. ${ }^{22}$ In quest'ultima versione, il saggio tiene ampio conto anche della lirica antica. Culler non si occupa, dichiaratamente $(2001,152)$, dell'indirizzarsi della voce del poeta a un ricettore empirico, ma dell'appello a elementi della natura inanimata, a oggetti, o ad animali, o ad astrazioni, da parte di una voce poetica che proprio attraverso queste apostrofi impossibili afferma la sua autonomia e potenza creativa, e anche profetica, e pone in essere una temporalità separata, un presente lirico in cui si colloca l'enunciazione, la quale, attraverso l'apostrofe, si propone al lettore come un evento, e non, ad esempio, come una meditazione, o una rappresentazione. Questo fenomeno è evidentemente molto diverso dai tanti casi che incontriamo in Orazio di allocuzione come ammonizione, consolazione, persuasione a un interlocutore, nel quadro di una occasione comunicativa dal profilo riconoscibile. ${ }^{23}$ Il rifiuto di Culler di prendere in considerazione la specificità di questa situazione emerge ancor più chiaramente nella rielaborazione recente del suo saggio, ove proprio nel caso di Orazio egli propone un conteggio delle diverse tipologie di interlocuzione (carmi rivolti a persone presunte reali, a persone presunte fittizie, a divinità, a oggetti), ma le riconduce infine tutte all'unica funzione di espediente artistico, ogni volta privo di rilevante connessione specifica con la condizione dell'interlocutore. ${ }^{24}$ Già Heinze, cui anche Culler si richiama, non distingueva i casi in cui il ricettore dell'allocuzione è persona reale, e dunque possibile destinatario di una comunicazione verbale, volta, idealmente o realmente, a condizionarne la volontà, dai casi in cui si tratta di una divinità o di un oggetto inanimato, che non può esserne destinatario, se non su un piano meramente fantastico, e in cui il condizionamento della volontà non può trovare effettivo spazio.

La distinzione è però, a mio giudizio, molto rilevante, come ho avuto modo di affermare in altre occasioni. ${ }^{25}$ L'indirizzarsi della voce lirica oraziana verso una seconda persona può essere considerata come un fenomeno unitario, indipendente dalla natura dell'interlocutore, se ci si attiene soltanto all'aspetto formale del fenomeno, se cioè lo consideriamo soltanto come una modalità costruttiva che conferisce al carme, e alla voce lirica stessa, una dimensione temporale, spaziale e una risonanza diversa da quelle che avrebbe una voce isolata. Heinze vedeva in questa modalità un ostacolo all'espressione della soggettività, e la valutava perciò negativamente. Culler vi vede, in positivo, un procedimento artistico ricco di effetti e di risonanze di grande portata. Queste due opposte letture del fenomeno allocutivo hanno tra loro in comune la scelta di considerarlo in quanto qualità 'interna' del testo, senza tenere conto del ponte verso la realtà esterna che si determina quando il testo nomina come proprio interlocutore un personaggio contemporaneo riconoscibile come reale. Questo approccio 'interno' è del resto condiviso da quasi tutti coloro che, sulla scia di Heinze, si sono occupati della questione. Un ponte verso la dimensione esterna, e biografica, è però contemplato da Alessandro Barchesi, secondo cui leggendo il testo lirico siamo indotti a ricostruire le condizioni originarie della sua composizione, e va ammessa la possibilità che una corretta interpretazione di un carme includa la memoria della situazione biografica dell'autore.$^{26}$ Soprattutto Michèle Lowrie, che ha avuto il maggior merito nel rilanciare negli studi oraziani il dibattito teorico sulla natura del discorso lirico confrontandosi 
con le più recenti elaborazioni di teoria della letteratura, ha mostrato disponibilità, $\mathrm{e}$ interesse, a prendere in considerazione il rapporto tra la dimensione propriamente estetica del testo e la dimensione esterna, interrogandosi con impegno sugli effetti che non tanto i contenuti, quanto la stessa qualità estetica del discorso possono avere sul piano della realtà. La sua conclusione è aperta: il testo stesso delle odi ci guiderebbe a operare, con la nostra immaginazione di lettori, un andiriveni dall'interno all'esterno del testo, seguendo una dicotomia che è iscritta nel testo. ${ }^{27}$

Da parte mia non nego certo la validità di una impostazione orientata verso il funzionamento 'interno' del procedimento allocutivo, e concordo, come la stessa Michèle Lowrie, con la formulazione di Alessandro Barchiesi secondo cui Orazio, muovendo dalla condizione della ricezione della lirica greca, nella quale l'occasione originale è un tratto dominante, trasforma l'occasione in un elemento tematico interno alla sua lirica. ${ }^{28}$ Credo però che nei molti casi in cui la persona alla quale la voce lirica si rivolge è una figura contemporanea nota, ben riconoscibile nella sua identità per $\mathrm{i}$ contemporanei, al punto che per lo più è ancora riconoscibile per noi, agli effetti che la 'forma del tu' ha in ogni caso sul piano artistico ed espressivo si aggiungono e si integrano altri effetti, di ordine in parte diverso, di cui credo si debba tenere conto in sede di interpretazione: il riferimento del testo a un ambito di relazioni personali reali del poeta genera un effetto di concretezza dell'esperienza da cui nasce, o simula di nascere, la voce lirica. ${ }^{29} \mathrm{E}$, soprattutto, l'indirizzarsi della voce lirica a persone determinate ritaglia un ambito di ricezione separato da quello del lettore generico (l'interlocutore stesso, l'ambiente delle relazioni personali dell'interlocutore). Non lo definirei un ambito privilegiato, perché non dubito, e non ho mai dubitato, che per Orazio l'ambito di destinazione privilegiato fosse sempre e comunque quello della generalità dei lettori e dei posteri, ma un ambito separato rispetto al quale il lettore generico, vero destinatario, è indotto a confrontarsi. Ritengo anche che la frequenza con cui nella raccolta lirica oraziana incontriamo l'interlocuzione con personaggi reali del suo ambiente di relazioni private sia legata al contesto reale della produzione e della circolazione della sua poesia, che pur destinandosi alla generalità dei lettori, nasce e circola in prima istanza in una dimensione più ristretta in cui molto contano le relazioni personali che la 'forma del tu' viene a coinvolgere. Credo dunque che la 'forma del tu' non sia solo una eredità inerte della modalità performativa della lirica greca arcaica, non sia solo 'forma', ma abbia una sua vitalità attuale per Orazio, in quanto corrisponde a una effettiva dinamica della genesi e della comunicazione dei testi entro un quadro di relazioni personali, e di occasioni, che egli propone al lettore generico e ai posteri entro la costruzione letteraria dei suoi testi lirici. ${ }^{30}$

Considerazioni analoghe si potrebbero fare anche per non poca parte della lirica moderna. Nel quadro delle discussioni suscitate dalle pagine di Culler sull'apostrofe, Paul Alpers ha rilevato che prima del romanticismo il carattere occasionale era condizione ordinaria della lirica, e che la funzione occasionale poteva comportare un andamento allocutivo connesso alle effettive funzioni di raccomandazione, consolazione ecc., le quali condizionano strettamente l'atteggiarsi della voce lirica e il gioco stesso delle apostrofi. ${ }^{31}$

3. Se deve essere sfumata, e problematizzata, la contrapposizione tra lirica antica e lirica moderna sulla base della presenza delle forme allocutive e delle loro funzioni, tanto più deve essere sfumata e problematizzata la contrapposizione tra lirica greca nella fase orale e lirica latina (ma anche ellenistica) destinata a fruizione libraria per 
quanto riguarda il rapporto tra testo e occasione. In anni recenti tra i grecisti si è venuta consolidando la convinzione che anche nella fase più propriamente orale della cultura greca le esecuzioni dei testi lirici non potevano essere sempre realizzate davanti all'uditorio stesso che il testo propone come interlocutore del suo messaggio. Il rapporto diretto e 'naturale' tra occasione comunitaria, genesi del canto e sua esecuzione non appare più come condizione strutturale costante della lirica greca arcaica, ${ }^{32}$ anche se non si può dubitare della intensa integrazione della lirica arcaica col tessuto della comunità. Oggi i grecisti giustamente usano valorizzare il momento della re-performance, la replica dell'esecuzione del canto, che lo distaccava necessariamente dalla situazione in cui esso si era originato. ${ }^{33}$ Ma non si tratta solo di questo. ${ }^{34}$ Appelli all'intera cittadinanza potevano essere eseguiti davanti a un ristretto uditorio simposiale già fin dalla prima esecuzione, assumendo quindi, fin dalle origini, e certo abitualmente in esecuzioni successive, una dimensione apertamente fittizia. Proprio il simposio doveva essere sede, oltre che di prime esecuzioni, soprattutto di ri-esecuzioni in cui l'uditorio doveva essere abituato a sentire apostrofi e allocuzioni non direttamente a lui rivolte, ma avvertite come fittizie in quella sede, anche se riferibili a funzioni comunicative occasionali determinate: in alcuni casi reali e realizzate in passato, ma in altri casi soltanto possibili nel passato, o realizzabili in futuro. In tutti i casi, certo comunissimi, e probabilmente prevalenti, di ri-esecuzioni, l'uditorio simposiale doveva essere abituato, come noi, a ricostruire l'occasione dell'esecuzione originale attraverso estrapolazioni congetturali sulla base del testo stesso, e, come noi, non poteva sapere se quella esecuzione originale si fosse realmente svolta nell'occasione presupposta dal testo, davanti al destinatario presupposto dal testo, o se si trattasse invece di una occasione meramente ideale, fittizia: perché non si può dubitare che nel simposio fossero abituali anche prime esecuzioni di canti d'amore rivolti a persone assenti, o di carmi politici rivolti a tutta la cittadinanza. L'uditorio antico della poesia orale, dunque, veniva spesso a trovarsi in una condizione paragonabile a quella del lettore moderno di quei testi: nella condizione cioè di dover congetturare sulla base del testo stesso situazioni occasionali della sua genesi delle quali non poteva conoscere la realtà e consistenza, che a volte sarà stata effettiva, a volte fittizia.

Ci è impossibile determinare, volta per volta, nei diversi autori e nei diversi componimenti, in quali misure e modalità, diverse in tempi e circostanze diverse, operasse nella comunicazione del testo, e nel suo effetto sul piano del coinvolgimento estetico ed emotivo del pubblico, l'eventuale scarto comunicativo determinato dalla finzione testuale di un'allocuzione rivolta a uditorii diversi da quello presente alla performance, o lo scarto comunicativo determinato dalla allocuzione all'uditorio di una originaria performance, connessa a una specifica occasione reale, diverso dall'uditorio della re-performance attuale. Uno scarto comunicativo ogni volta in certa misura analogo a quello che il testo proponeva e propone al lettore antico e moderno, che fruiva/fruisce il testo lirico in forma scritta trovandovi un 'tu' in cui, caso per caso, non sa se e fino a che punto ha diritto di identificarsi e che si trova dunque indotto a ricostruire, sulla base delle indicazioni del testo, la situazione comunicativa, reale o fittizia, che motiva l'allocuzione..$^{35}$

La coscienza di queste molteplici possibilità di destinazione (occasione della prima esecuzione, ri-esecuzioni, lettura), e del loro eventuale sovrapporsi nell'esperienza dei fruitori, poteva essere presente al poeta fin dalla originaria concezione del suo testo. 
L'abitudine a fruire il testo lirico attraverso la lettura è iniziata almeno nel V secolo, e probabilmente da prima, ma ciò che conta in ottica romana è che comunque fin dalla prima età ellenistica, pur nel perdurare delle esecuzioni di poesia lirica in situazioni simposiache e religiose, la lettura era sicuramente il principale se non unico modo di accesso alla lirica arcaica. E il lettore greco doveva essere ben avvezzo a cercare di ricreare la situazione occasionale originaria presupposta dai testi sulla base del testo stesso, senza sapere se si trattasse di una occasione reale o fittizia e convenzionale. I poeti romani scrissero le loro liriche richiamandosi a un corpus di liriche greche molto lontane nel tempo che per lo stesso pubblico greco erano fruibili da gran tempo solo, o quasi solo, in forma scritta, e che ogni volta richiedevano la ricostruzione, sulla base di un testo scritto, di una originaria situazione che motivasse la forma allocutiva, oltre che molti aspetti del significato e della forma stessa.

I testi dei lirici greci disponibili agli editori alessandrini si erano tramandati nel tempo in quanto erano stati ritenuti meritevoli di essere rieseguiti oralmente davanti a uditorii diversi, e di essere riproposti in forma scritta ai lettori di nuove età. Ciò presuppone che questi testi avessero un interesse generale che va ben oltre quello legato alla situazione occasionale originaria. ${ }^{36} \mathrm{E}$ questo interesse generale era la ragione della loro sopravvivenza. Ma che la comprensione del loro messaggio risultasse allora, e risulterebbe oggi, precisata e arricchita della conoscenza della loro originaria destinazione occasionale sarebbe difficile negare.

L'opposizione tra pubblico specifico, direttamente partecipe dell'occasione, nella fase orale, e pubblico presunto come universale, estraneo all'occasione, nella fase libraria, va sfumata anche su questo secondo versante, il versante della universalità della destinazione libraria. La prevalenza della dimensione libraria della comunicazione dei generi non teatrali a partire dall'età ellenistica e in particolare a Roma fin dall'inizio della produzione letteraria formale rendeva certo fondamentale la coscienza di una potenzialità di destinazione universale del messaggio letterario. Ma non possiamo dimenticare le condizioni di fatto della circolazione del libro antico, e dobbiamo tenere conto di come esse si dovessero riflettere sull'idea di pubblico degli autori stessi. A Roma la produzione artigianale e commerciale destinata al pubblico generico ha una consistente presenza al tempo di Cicerone e si sviluppa alquanto in età augustea, ma convive costantemente con le mai interrotte prassi della produzione domestica e della diffusione privata entro cerchie di amici. La dimensione di fatto ristretta della destinazione libraria, e le molteplici occasioni sociali, anch'esse per lo più in sedi ristrette e private, di esecuzione orale in anteprima dei testi destinati alla pubblicazione libraria e di presentazione privata di copie di dedica dei testi pubblicati sono condizioni stabili e strutturali che collocano i testi augustei, pur idealmente proiettati verso un pubblico universale, entro una dimensione molto vicina all'autore e al suo ambiente. ${ }^{37}$ Una dimensione nella quale i lettori di cui l'autore ha più diretta percezione, i lettori che faranno sentire il loro consenso o dissenso sull'opera, sono spesso le stesse cerchie di persone coinvolte dalle occasioni presupposte dal componimento lirico. Pur trattandosi di poesia destinata alla lettura, e proiettata idealmente verso una destinazione universale, è poesia che di fatto ha come più immediato e diretto pubblico gruppi limitati di lettori e ascoltatori, partecipi, più o meno diretti, delle occasioni che hanno suggerito le parole di raccomandazione, ammonimento, conforto, elogio, celebrazione nelle molte odi che coinvolgono, nell'occasione presupposta, personaggi reali della cerchia del poeta. 
29 4. Il nodo di fondo resta però aperto. E consiste nella legittimità del passaggio dall'occasione quale è presupposta dal testo, nella sua dimensione letteraria, e dunque testuale, ed estetica, e perciò stesso fittizia, alla occasione intesa come realtà storica e biografica.

30 Le ragioni che oggi portano ad attribuire nuova rilevanza al rapporto del testo con $\mathrm{i}$ referenti reali sono molteplici. Sono connesse a un generale atteggiamento neostoricistico, che riassegna alla letteratura un forte radicamento nel contesto sociale e valoriale del suo tempo, e un ruolo privilegiato di testimonianza su mentalità e valori. Di qui la grande rilevanza della utilizzazione della letteratura e della analisi dei testi letterari nel vasto quadro dei cultural studies. Negli studi sulla poesia latina si è anche assistito a una sorta di emulazione con i colleghi grecisti, e a una forzosa estensione ad essa della dimensione orale della comunicazione. Proprio mentre tra i grecisti si veniva sviluppando la consapevolezza del carattere fittizio di molta parte delle allocuzioni all'uditorio nella lirica orale, e della vasta realtà delle ri-esecuzioni e della stessa lettura in cui questa finzionalità diventava strutturale, presso i latinisti si è cercato di valorizzare la dimensione della comunicazione orale a Roma, così da recuperare per questa via un legame vivo e diretto tra testo e società, tra testo e occasione. ${ }^{38}$ Varie proposte in questo senso sono state avanzate, con molto impegno, in anni recenti. Mi riferisco all'audace e discusso volume di Thomas Habinek, The World of Roman Song, che cerca di radicare le espressioni letterarie latine entro un contesto di rituali in cui la dimensione orale ha un ruolo primario, ma anche al recentissimo The Roman Audience di T.P. Wiseman, che ricompone l'intero sviluppo della letteratura latina entro una dimensione di comunicazione orale, ponendosi a coronamento, per così dire, di una serie non certo esile di lavori, iniziata almeno a partire da un lavoro di Kenneth Quinn del 1982, che hanno cercato, con maggiore o minore decisione, di attribuire priorità al momento della dimensione orale nella fruizione della letteratura a Roma. ${ }^{39}$

31 Questi studi hanno avuto ed hanno, in vario modo, il merito di dare evidenza al momento orale della comunicazione della letteratura a Roma, spesso trascurato in passato, oltre al merito, dal mio punto di vista, di impegnarsi a connettere la produzione e la fruizione della letteratura al contesto sociale, ma non riescono, a mio giudizio, a convincerci che la poesia a Roma viva prioritariamente come espressione orale diretta, generata e comunicata nell'immediatezza delle situazioni occasionali o rituali. Una compiuta discussione di questa impostazione richiederebbe troppo spazio e ci porterebbe fuori tema: posso limitarmi a rinviare a un contributo di Holt N. Parker il quale ne ha condotto una confutazione efficacissima, che da parte mia condivido in pieno, e ha argomentato in modo stringente la tesi tradizionale secondo cui la comunicazione della letteratura a Roma ha carattere essenzialmente scritto e librario. I testi poetici, certo, venivano spesso recitati, ma solitamente davanti a uditorii ristretti, prevalentemente di persone che facevano più o meno direttamente parte delle cerchie di relazioni personali dell'autore, molto più raramente davanti a ampi uditorii pubblici e, ciò che più conta, le esecuzioni erano comunque considerate come dice giustamente Parker, "preparatorie, ancillari, o supplementari, rispetto alla situazione ordinaria, e non marcata, della lettura personale". ${ }^{40}$

32 La lirica di Orazio, come già quella di Catullo è dunque, io credo, destinata alla lettura e a una finalità primariamente estetica. Salvo l'ovvia eccezione del Carmen saeculare, eseguito da un coro e concepito e destinato, sia pur come oggetto estetico, a essere parte di un rito. In forma scritta, in età augustea i testi continuavano a circolare 
prevalentemente in ambiti relativamente ristretti, non popolari, sia pure nella prospettiva ideale di un pubblico universale. Nel caso dei molti carmi che coinvolgevano persone reali della cerchia del poeta in relazione a una occasione che richiedeva consolazione, elogio, raccomandazione, ringraziamento ecc. è molto probabile che i testi venissero fatti conoscere, separatamente, alle persone coinvolte prima della pubblicazione in libro, con invio del testo scritto o tramite recitazioni. E comunque, anche se lette all'interno del libro, le odi rivolte a persone reali instaurano con esse un rapporto comunicativo particolare che, come abbiamo detto sopra, si integra con quello rivolto alla generalità dei lettori ma non coincide con esso. Nei confronti di questi dedicatari, e di quanti erano personalmente coinvolti dall'occasione presupposta dal testo, sia la comunicazione in anteprima, sia la pubblicazione libraria, rappresentavano una sorta di celebrazione letteraria dell'occasione originaria. Non un invito, o una consolazione, o un elogio quale quello che si fa nelle relazioni sociali comuni con il linguaggio comune, con gli atti linguistici nel senso corrente del concetto, o con comunicazioni epistolari, ma una trasposizione nella dimensione fittizia della letteratura di esperienze che nella realtà extra-letteraria coinvolgono il poeta, uno o più destinatari con cui egli intrattiene una relazione personale, una cerchia di relazioni private intorno al poeta e ai destinatari individuali. Il testo scritto con cui si attua questa trasposizione letteraria dell'esperienza occasionale si rivolge, al tempo stesso, a un pubblico generico, e universale, al cui cospetto, idealmente, si compie questa celebrazione letteraria di occasioni determinate. Si tratta del resto di situazioni riconducibili a una ristretta serie di 'occasione-tipo' atte a rappresentare una modalità di rapporti al tempo stesso personali e riferibili all'esperienza sociale di tutti i lettori. ${ }^{41}$

La dimensione biografica e quella letteraria devono essere tenute distinte, ma non sono tra loro irrelate. Sarebbe ingenuo, e anzi senz'altro ridicolo, pensare che le odi di invito di Catullo o di Orazio potessero servire come biglietti di invito: ma queste eleganti variazioni di un tipo poetico tradizionale trasferiscono sul piano della letteratura, e propongono alla generalità dei lettori, esperienze amicali reali, tra personaggi noti alla generalità del pubblico, e in vari casi contengono riferimenti a situazioni specifiche, certamente biografiche, che conferiscono un senso particolare al componimento: l'applauso della folla a Mecenate $(1,20,5-8)$ non può essere invenzione letteraria, e non lo sarà lo scampato pericolo da parte del poeta $(3,8,6-8)$ o il rientro in patria del compagno d'armi a Filippi $(2,7)$ che hanno dato occasione, e un significato ogni volta diverso, a quel particolare carme di invito. Sarebbe difficile sostenere che l'identificazione delle circostanze occasionali che carmi come questi trasferiscono sul piano della finzione letteraria è irrilevante per la comprensione dell'ode. Nel caso di 1, 20 , il testo parla soltanto di applauso a teatro. Ma Fraenkel stesso non si accontenta di ciò che si ricava dal testo e, alla fine della sua analisi, riferisce l'episodio dell'applauso alla guarigione di Mecenate da una grave malattia (p. 216 s.), recuperando l'informazione da un altro testo, l'ode 2, 17 nella quale il riferimento alla malattia non è esplicito, come ammette lo stesso Fraenkel, mentre è esplicitato da Porfirione. È il procedimento che solo due pagine prima Fraenkel aveva ribadito come illegittimo, appunto in riferimento a chi volesse andare a cercare al di fuori di quest'ode dettagli sulle sue circostanze occasionali. Fraenkel giustamente riconosce in quest'ode un vero Gelegenheitsgedicht, suscitato da una occasione specifica e scritto per essa, ma ritiene che il suo significato più rilevante sia nella contrapposizione simbolica finale tra i vini di diversa qualità in quanto emblemi della sua scelta di vita, e cerca così di scindere il vero significato dell'ode dal dato occasionale esterno. Io credo che nelle intenzioni 
dell'autore, se mai le potessimo ricostruire, e nell'esperienza dei lettori antichi, se mai la potessimo ricostruire, l'ode fosse, come è ora per noi, più significativa come trasposizione letteraria di una esperienza di relazione interpersonale di amicizia e affetto, una trasposizione che, pur perfettamente fruibile da ogni lettore esterno, poteva risultare più ricca di sfumature e contenuti per quanti avevano contezza dell'occasione che aveva generato la composizione e potevano in qualche misura confrontare forma, tono e linguaggio di questo carme, nella sua dimensione estetica, con forma, toni e linguaggio propri di questa e di analoghe relazione interpersonali nella loro dimensione sociale e biografica. Ricavare da fonti esterne al testo elementi contestuali certi che consentano di avere una migliore percezione del profilo reale dei personaggi cui la voce poetica è rivolta e delle situazioni presupposte dal testo può avere per questo, io credo, un'effettiva incidenza sull'interpretazione del testo nella sua dimensione estetica.

\section{BIBLIOGRAFIA}

Alpers, P., 2013, Apostrophe and the Rhetoric of Renaissance Lyric, "Representations" 122, 1-22.

Barchiesi, A., 2000: Rituals in Ink: Horace on the Greek Lyric Tradition, in M. Depew, D. Obbink (eds.), Matrices of Genre. Authors, Canons and Society, Cambridge Mass. - London, 167-182 (rist. in Lowrie (ed.) 2009, 418-440).

Barchiesi, A., 2001: The Crossing, in S.J. Harrison (ed.), Texts, Ideas, and the Classics. Scholarship, Theory, and Classical Literature, Oxford, 142-163.

Barchiesi, A., 2007: Carmina: Odes and Carmen Saeculare, in S. Harrison (ed.), The Cambridge Companion to Horace, Cambridge, 144-161.

Barchiesi, A., 2009: Lyric in Rome, in Budelmann (ed.) 2009, 319-335.

Becker, C., 1959: recensione a Fraenkel 1957, “Gnomon” 31, 592-612.

Budelmann, F., 2009, Introducing Greek Lyric, in Budelmann (ed.) 2009, 1-18.

Budelmann, F. (ed.), 2009, The Cambridge Companion to Greek Lyric, Cambridge.

Burnett, A.P., 1983: Three Archaic Poets: Archilochus, Alcaeus, Sappho, London.

Buttmann, Ph., 1828: Mythologus oder gesammelte Abhandlungen über die Sagen des Altertums I, Nebst einem Anhang über das Geschichtliche und die Anspielungen im Horaz, Berlin.

Carey, Ch., 2009: Genre, Occasion and Performance, in Budelmann (ed.) 2009, 21-38.

Citroni, M., 1995: Poesia e lettori in Roma antica. Forme della comunicazione letteraria, Roma - Bari.

Citroni, M., 2015: Edito e inedito, pubblico e privato: Marziale, Stazio e la circolazione dei testi scritti in età flavia, "Segno e testo" 13, 89-123.

Commager, S., 1962: The Odes of Horace. A Critical Study, New Haven - London 1962.

Copley, F.O., 1962: Yet once more... (recensione a Commager 1962), “Arion” 1, 4, 84-88. 
Culler, J., 2001: The Pursuit of Signs. Semiotics, Literature, Deconstruction. With a new preface by the author, London - Neew York (first ed. London 1981).

Culler, J., 2015: Theory of the Lyric, Cambridge Mass. - London.

Cunningham, M.P., 1959: recensione a Fraenkel 1957, “CPh” 54, 127-128.

Davis, G., 1991: Polyhymnia. The Rhetoric of Horatian Lyric Discourse, Berkeley - Los Angeles. de Man, P., 1985: The Lyrical Voice in Contemporary Theory, in Ch. Hošek, P. Parker (edd.), Lyric Poetry: Beyond New Criticism, Ithaca.

Edmunds, L., 2001: Intertextuality and the Reading of Roman Poetry, Baltimore - London 2001.

Farrell, J., 2005: Eduard Fraenkel on Horace and Servius, or, Text, Contexts, and the Field of "Latin Studies", “TAPhA” 135, 91-102.

Feeney, D., 1993: Horace and the Greek Lyric Poets, in N. Rudd. (ed.), Horace 2000: a Celebration. Essays for the Bimillennium, London, 41-63 [= Lowrie 2009, 202-231].

Fowler, D., 1993: Images of Horace in Twentieth Century Scholarship, in Ch. Martindale, D. Hopkins (eds.), Horace Made New. Horatian influences on British writing from the Renaissance to the twentieth century, Cambridge, 268-276.

Fraenkel, E., 1957: Horace, Oxford.

Habinek, Th.N., 1992: Grecian Wonders and Roman Woe: The Romantic Rejection of Rome and its Consequences for the Study of Latin Literature, in K. Galinsky (ed.), The Interpretation of Roman Poetry: Empiricism or Hermeneutics?, Frankfurt a. M. - Bern - New York - Paris 1992, 227-242.

Habinek, Th., 2005: The World of Roman Song. From Ritualized Speech to Social Order, Baltimore London.

Harrison, S.J., 1995: Some Twentieth-century Views of Horace, in S.J. Harrison (ed.), Homage to Horace. A Bimillenary Celebration, Oxford, 1-16.

Heinze, R., 1923: Die horazische Ode, "Neue Jahrb." 51, 153-168, rist. in Vom Geist des Römertums, Stuttgart $1960^{3}$ (rist. Darmstadt 1972), 172-189; trad. italiana in Santini 2001, 55-74; trad. inglese in Lowrie (ed.) 2009, 11-32.

Klingner, F., 1958: recensione a Fraenkel 1957, “JRS” 48, 170-178.

Leeman, A.D., 1958: Some Comments on Fraenkel's Horace, “Mnemosyne” 11, 244-250.

Lowrie, M., 1997: Horace's Narrative Odes, Oxford.

Lowrie, M., 2009: Writing, Performance, and Authority in Augustan Rome, Oxford.

Lowrie, M. (ed.), 2009: Oxford Readings in Classical Studies. Horace: Odes and Epodes, Oxford.

Miller, P.A., 1994 Lyric Texts and Lyric Consciousness, London New York.

Mommsen, Th., 1905 Die Akten zu dem Säkulargedicht des Horaz, in Th. Mommsen, Rede und Aufsätze, Berlin, 351-359.

Nagy, G., 1990, Pindar's Homer. The Lyric Possession of an Epic Past, Baltimore - London.

Parker, H.N., 2009: Books and Reading Latin Poetry, in W.A. Johnson, H.N. Parker (a cura di), Ancient Literacies, Oxford, 186-229.

Quinn, K., 1982: The Poet and his Audience in Augustan Age, ANRW II 30, 1, Berlin - New York, 75-180. 
Reitzenstein, R., 1924: Eine neue Auffassung der horazischen Ode, "Neue Jahrbb. " 53, 232-241 (= id., Aufsätze zu Horaz, Darmstadt 1963, 73-82).

Rudd, N. ,1958: Professor Fraenkel's Horace, "Hermathena" 91, 43-54.

Santini, C., 2001: Heinze e il suo saggio sull'ode oraziana, Napoli.

Schmidt, P.L., 1985 Horaz Säculargedicht- Ein Prozessionslied?, “AU” 28 (4), 42-53 (trad. inglese in Lowrie 2009, 122-140).

Silk, E.T., 1964: recensione a Commager 1962, “AJPh” 85, 311-314.

Syndikus, H.P., 1972: Die Lyrik des Horaz. Eine Interpretation der Oden, I, Darmstadt.

Sokolov, R.A., jr. 1962: recensione a Commager 1962, “The Harvard Crimson” 14.05.1962.

Vendler, H., 1997: Poems, Poets, Poetry: an Introduction and Anthology, Boston Mass.

Wiseman, T.P., 2015: The Roman Audience. Classical Literature as Social History, Oxford.

\section{NOTE}

1. Fraenkel 1957, 26. Il principio era ribadito con non minore energia a p. 73 s. e p. 208 s., e veniva più volte richiamato nel corso della trattazione: cfr. pp. 63 n. 1; 154; 163 e n. 5; 214 s.; 313 s.; 370 n. 1. Fraenkel gli attribuiva tanta importanza da farne una voce del suo pur molto selettivo indice degli argomenti (p. 458: "Poems, understanding of, non dependent on outside information", voce che peraltro non rinvia a tutti i passi rilevanti).

2. Sokolov 1962: "the classical world is ... the last field for which the New Criticism is still new".

3. Così Copley 1962, 85 e Silk, 1964, 311. L'influenza del New Criticism sul volume oraziano di Commager è segnalata anche da Habinek 1992, 237 s.; Harrison 1995, 8; Lowrie 1997, 6.

4. Lo rilevava anche Fowler 1993, 273.

5. Fowler 1993, 273 s.; Farrell 2005, 92 s., il quale ritiene però che la condivisione di quel principio determini comunque un'effettiva affinità dell'approccio di Fraenkel a quello degli allora nuovi formalismi. È chiaro che Fraenkel, pur cercando di valorizzare le qualità proprie dei componimenti considerati, interpreta complessivamente l'opera di Orazio nel quadro della situazione politica e culturale augustea, nonché nel quadro delle tradizioni letterarie familiari a lui e al suo pubblico. Qualche singolo caso in cui Fraenkel stesso finisce col far dipendere l'interpretazione di un carme da informazioni esterne ad esso è segnalato da Rudd 1958, 47 (entro una vivace contestazione di questo principio fraenkeliano); Becker 1959, 609 s. e cfr. 597; Farrell 2005, 94; Lowrie 1997, 6. Cfr. anche quanto dirò più oltre a proposito di carm. 1, 20.

6. Cunningham 1959, 128.

7. Leeman 1958, 246.

8. Il passo di Schlegel (Lezioni di arte drammatica e letteratura, cap. 26) cui Fowler allude è del tutto calzante nella formulazione ("un'opera d'arte deve contenere in sé tutto ciò che è necessario alla sua piena comprensione"), ma si riferisce a una situazione specifica e in parte diversa: la comprensibilità dei riferimenti storici nella poesia drammatica, e in particolare nel dramma storico, per il pubblico teatrale di epoche diverse da quella in cui il testo è stato inizialmente prodotto. La nota frase di Coleridge (Biographia literaria, cap. 14) secondo cui "nothing can permanently please which does not contain in itself the reason why it is so, and not otherwise" (citato da Fowler 1993, 310 n. 26) si riferisce all'intrinseca necessità, in un'opera, di una certa forma poetica, metrica, linguistica e di un certo contenuto, negando che uno di questi elementi possa mancare o essere diversamente da come è. 
9. Cfr. ad es. Estetica, ed. ital. a c. di N. Merker, Torino 1972, p. 1251 s. (parte 3, sez. 3, cap. 3, C, II $1, \mathrm{~b}, \mathrm{~B}, \mathrm{AA})$ "se l'opera d'arte lirica non deve cadere nella dipendenza dall'occasione esterna e dai fini in essa contenuti, bensì sussistere per sé come un tutto autonomo, allora è essenziale che il poeta si serva della circostanza appunto solo come occasione per esprimere se stesso, il suo stato d'animo ... il poeta lirico ... per quanto variamente mescoli il suo interno con il mondo esistente e le condizioni, gli intrecci e i destini di esso, tuttavia palesa, nel manifestare questo argomento, solo la vitalità autonoma dei propri sentimenti e delle proprie considerazioni" (trad. di N. Merker e N. Vaccaro).

10. Cfr. Buttmann 1828, 303 s. e 316 ove è più volte ripetuto che le odi di Orazio vanno intese "bloss idealisch", che il mondo che esse creano è un mondo di "poetische Ideen" e non di fatti storci. Cfr. anche p. 311: in Orazio argomenti e persone sono creazioni dell'artista come le immagini e le parole. Va però detto che Buttmann non ammette questo carattere meramente ideale per le odi civili.

11. Fraenkel 1957, 41. Anche questo elemento era in Buttmann 1928, 315 s., ma si fondava su un presupposto diverso: non il dato storico 'oggettivo' del passaggio dalla comunicazione orale a quella libraria, ma l'intenzione di Orazio di porsi come poeta nazionale e di realizzare dunque carmi che fossero "von allen Seiten abgerundete Kunstwerke" (Fraenkel usa frequentemente "self-contained"), comprensibili per i contemporanei e i posteri.

12. Fraenkel 1957, 37, n. 1, e cfr. 38, nn. 1-4, ove sono citati, per la connessione in Grecia tra letteratura e vita della comunità, la Geschichte der griechischen Litteratur di K.O. Müller (la cui prima edizione, postuma, è del 1841), Epigramm und Skolion di R. Reitzenstein (Giessen 1893); Aristoteles und Athen (Berlin 1893) e Die griechische Literatur des Altertums (Berlin - Leipzig 1905) di Wilamowitz.

13. La parte avuta dall'entusiasmo per gli studi oralistici nel rilanciare i pregiudizi romantici sulla autenticità e vitalità della letteratura romana è ben individuata da Lowrie 2009, 9-13 che cita anche in proposito una brillante pagina di Feeney in TLS 28.4.2000, 9. Cfr. anche più oltre, n. 38.

14. Miller 1994.

15. Fraenkel 1957, 364-382 e cfr. 404 s. Questo aspetto della lettura di Fraenkel del carme secolare aveva subito suscitato le obiezioni di alcuni recensori pur molto elogiativi nei confronti dell' Horace: cfr. Klingner 1958, 176; Becker 1959, 607 s. Più articolate obiezioni, nello stesso senso, in Schmidt 1985, 45-47 [trad. ingl. in Lowrie (ed.) 2009, 128-130]. Barchiesi 2000, 179 s. [= Lowrie (ed.) 2009, 436] osserva acutamente come la valutazione di Fraenkel del carmen saeculare, ammirato proprio perché svincolato dalle esigenze del rito, riveli che per lui il legame col rito è ragione di autenticità e di positivo valore nel caso della poesia greca, e viceversa di fredda cerimonialità, e dunque di carenza di valore, nel caso della poesia latina: un presupposto (romantico) sotteso anche alla opposta valutazione di Mommsen 1905 [1891], che vedeva la debolezza estetica del carmen saeculare nell'essere troppo legato al rito. Aggiungiamo che Mommsen a p. 358 indicava come difetto del carme anche il fatto che, a suo parere, almeno un importante passaggio risultava comprensibile solo ai partecipanti al rito o a chi aveva a disposizione informazioni esterne al testo stesso sullo svolgimento del rito (appunto le informazioni a noi disponibili solo grazie all'iscrizione scoperta nel 1890 e pubblicata dallo stesso Mommsen l'anno seguente), mentre il carme doveva essere inteso come rivolto a una vasta platea di lettori. Analogamente un passo di Friedrich Leo risalente al 1903 citato da Fraenkel 1957, 370, n. 1.

16. Culler 2015 offre un articolato ragguaglio critico sul dibattito teorico contemporaneo relativamente al presunto carattere fittizio dell'io lirico e al problema del suo rapporto con l'io dell'autore, nonché sul complessivo problema del rapporto finzione/realtà nella lirica. 
17. Vd. soprattutto Budelmann 2009, 10-17. In questo senso anche Barchiesi 2000, 170 s.; 175 [= Lowrie (ed.) 2009, 422-424; 430 s.]; Barchiesi 2001, 151-154; Barchiesi 2007, 156; 159; Lowrie 2009, $74 \mathrm{e} 82 \mathrm{~s}$. Cfr. anche più oltre, n. 32.

18. Di Heinze 1923 (rist. in Heinze 1960, da cui cito), e del suo impatto sugli studi oraziani mi sono occupato estesamente in Citroni 1995, 271-278 e 336-341. Sull'impatto anche Santini 2001, 40-54. In anni più recenti si sono confrontati col saggio di Heinze, tra gli altri, Lowrie 1997, spec. 19-24; Lowrie 2009, 63-117 (spec. 81-83); Barchiesi 2000, 173-176 [= Lowrie (ed.) 2009, 428-432]; Barchiesi 2007, 155-158 (e cfr. 150-153); Edmunds 2001, 83-94.

19. In Citroni 1995 davo conto delle due sole significative posizioni contrarie a Heinze espresse fino ad allora: quelle di Reitzenstein 1924 e Syndikus 1972, 14 s. Entrambi negano il ruolo costrittivo che avrebbe per Orazio il modello allocutivo della lirica greca, rivendicano un maggiore spazio al momento dell'introspezione nella lirica oraziana e contestano l'estensione alla totalità delle odi del presupposto della presenza dell'interlocutore. Syndikus nega la legittimità di ricostruire una situazione quando questa non sia esplicitamente indicata dal testo. Reitzenstein rilevava che l'allocuzione manca talvolta anche nella lirica greca, che il lettore romano era ben avvezzo a leggere poesia greca e romana rivolta a un interlocutore, e che in generale nella poesia antica non ha senso un'opposizione tra espressione della soggettività e comunicazione con gli altri. Da parte mia, condividendo tutte queste obiezioni, aggiungevo più radicali riserve, che brevemente preciserò nelle pagine seguenti, sull'impostazione formalistica dello studio di Heinze. Gli studiosi che hanno discusso il saggio di Heinze in anni più recenti (vd. sopra, n. 18) ne hanno largamente accettato la tesi, salvo rilevanti riserve da parte di Lowrie 2009, di cui diremo.

20. Heinze stesso (pp. 178-180) analizza alcuni esempi, interpretandoli, non senza qualche forzatura, ora come mere variazioni formali di lirica sostanzialmente monologica, ora come consapevoli imitazioni di Orazio e della sua caratteristica modalità lirica.

21. Nella rivista "Diacritics" 7 (4), 59-69. Ripreso in Culler 2001, 149-171 (=1981 1 , 135-154).

22. Culler 2015, 186-243.

23. Il punto è segnalato da Lowrie 1997, 21 n. 2., la quale però sembra attenersi alla stessa indistinzione, e considerare tutte le diverse forme di address in Orazio sostanzialmente sullo stesso piano, come elementi posti in essere dal testo (the Horatian addressee is ... as much a construct of the poetry as anything else...”. In Lowrie 2009 la posizione sarà in parte diversa, come diremo più oltre (e cfr. nn. 27 e 29).

24. Culler 2015, 201 s. Culler si richiama infatti a Davis 1991 e Edmunds 2001, due studi in cui con maggiore decisione viene sostenuto il carattere fittizio dell'occasione nei carmi oraziani, e comunque l'irrilevanza dei dati occasionali esterni ai fini dell'interpretazione. Cfr. Edmunds 2001,91 "the real addressee is a literary device".

25. Cfr. Citroni 1995, 272 s.; 338 s..

26. Barchiesi 2007, $150 \mathrm{~s}$. Vedi anche più oltre, n. 35.

27. Lowrie 2009, 117 definisce questa situazione come un'impasse, che alcuni testi ci consentirebbero però di superare (ma non precisa come). Alle pp. 66-71; 81-83; 101-103 la Lowrie pone il problema se al testo letterario possa essere attribuita l'efficacia operativa sulla realtà che è propria di un atto linguistico illocutivo. Giustamente lo nega in via di principio, perché la sua natura estetica separa il linguaggio letterario dall'operatività pragmatica del linguaggio comune. La Lowrie ammette però almeno la funzione di elogio e onore a un dedicatario. Io mi spingerei ad ammettere anche la possibilità di altre funzioni meno generiche di queste. Credo ad es. che un carme consolatorio, pur nella sua dimensione estetica, possa avere un certo ruolo di conforto verso la persona in lutto. Ma in ogni caso si tratta, eventualmente, della ricaduta pragmatica di un testo che ha natura essenzialmente estetica, e che opera con modalità diverse da quelle del linguaggio ordinario, come diremo anche più avanti. Oltre che di una funzione pragmatica di elogio, la Lowrie parla di altri e più ampi effetti esterni, di carattere essenzialmente metapoetico: 
un carme di invito quale 1, 20 non invita effettivamente un ospite (ovviamente!), ma interviene nel mondo provocando una riflessione sulla singolare situazione della poesia, collocata sia entro il mondo sia fuori di esso (p. 70); Orazio con la sua parola poetica, oltre a onorare le persone cui si rivolge, ottiene il risultato effettivo di rinegoziare le convenzioni del genere lirico e così di trasformarlo e reinventarlo, e anzi di rinegoziare la posizione della poesia nella società attraverso un ripetuto proporre e un ripetuto respingere il gesto dell'efficacia poetica sul mondo esterno (101-103). Temo però che questo tipo di interpretazioni metapoetiche rischino di trasferire entro il testo antico i nostri problemi di interpreti moderni.

28. Barchiesi 2000, 176 [= Lowrie (ed.) 2009, 431 s.] e cfr. Lowrie 2009, 101.

29. Lowrie 2009,67 , ammette che quando il testo è rivolto a una figura storica vivente diventa più arduo sostenere la distinzione tra ego lirico e autore e qualificare il carme come finzione.

30. Sviluppavo questo concetto in Citroni 1995, 336-341. Lowrie 2009, 102 pone la presentazione di se stesso e della sua rete sociale al mondo come uno degli effetti del direct address nelle odi di Orazio.

31. Alpers 2013, 18-20.

32. Lowrie 1997, 32 n. 31 lo definisce "un mito delle origini che, come tutti questi miti, dice solo una parte della verità". Una rivendicazione molto netta ed efficace del fatto che il poeta lirico arcaico era libero, come ogni poeta, di inventare sia il profilo dell'io che si esprime nel componimento sia le occasioni in cui tali espressioni si intendono formulate è in Burnett 1983, 3-7. Cfr. anche Feeney 1993, 55 s. [= Lowrie (ed.) 2009, 224 s.] sul persistere del pregiudizio romantico della superiorità rispetto a Orazio della lirica orale greca in ragione della sua spontaneità e autenticità, garantite dal radicamento sociale. Vd. anche Barchiesi 2007, 159. Lowrie 2009, 74 e 82 s., contrapponendosi a concetti correnti, e alla dicotomia posta da Heinze tra lirica greca e lirica romana, giustamente nega che riferimenti testuali a performance implichino necessariamente reale performance, che riferimenti testuali alla scrittura comportino separazione da un contesto sociale e proiezione verso la posterità, che la separazione da un contesto performativo o occasionale reale disancori la poesia da un organico rapporto con la società, che la performance reale comporti di per sé rapporto effettivo con la situazione presupposta dal testo eseguito, che la comunicazione poetica scritta priva di performance escluda efficacia performativa del testo.

33. Cfr. Nagy 1990, 80 s.; 342-344; 374; 412 s.; 432 s.; 437 e ad es. Budelmann 2009, 8.; Carey 2009, 34.

34. Sviluppo qui di seguito un concetto già proposto in Citroni 1995, $275 \mathrm{~s}$.

35. La lettura è intesa come re-performance ad es. in Nagy 1990, 171 con riferimento alla lirica greca e, sul piano della teoria generale della lirica, in Culler 2015, 138 s.; 186 s.; 226 e cfr. Vendler 1997, xli-xliii secondo cui "una lirica è un copione per la performance del suo lettore", il quale deve ricostruire la situazione in cui si colloca il parlante (ove situazione e persona parlante sono da considerare, per la Vendler, entrambi fittizi). Anche Barchiesi 2007, 150 s. (e cfr. Barchiesi 2000, 176 [= Lowrie (ed.) 2009, 432] e Barchiesi 2009, 332-334) considera ogni lettura di un testo lirico come una re-performance in cui il lettore è indotto a ricostruire le condizioni della performance originaria della voce lirica sulla base di una certa nozione che egli si fa dell'autore come fonte dell'enunciato: nozione che può collocarsi in una varietà di posizioni, dall'estremo di una voce del tutto generica a quello di una circostanziata specificità storico-biografica (vedi anche sopra, n. 26). La tesi, secondo cui "il principio generale di intellegibilità nella lirica dipende dalla fenomenalizzazione della voce poetica" (De Man 1985, 55) e dal riferimento dell'enunciato lirico a un personaggio fittiziamente creato è ampiamente contestata da Culler 2015, 35, 109-125 e passim, in quanto non applicabile a molta parte della lirica (moderna), ma è probabilmente valida per ampia parte della lirica antica. A p. 120 Culler approva la posizione di Barchiesi che ho qui riepilogata appunto in quanto ammette la possibilità di identificare nel carme sia una mera voce astratta sia la voce di un autore storicamente individuato anziché presumere sempre, 
secondo la concezione oggi prevalente, imitazione di un atto linguistico e dunque enunciazione di persona fittizia, in una situazione fittizia che deve essere ricostruita sulla base del testo.

36. Il principio è più volte affermato dagli studiosi di oralistica, che tendono a distaccare i testi dei lirici arcaici dalla soggettività particolare e privata dell'autore. Cfr. in proposito Miller 1994, 81; 88; 191.

37. Cfr. Citroni 2015.

38. In Lowrie 2009, 9-13 opportune osservazioni sulla ricerca di un recupero di autenticità nella poesia romana attraverso una forzosa applicazione ad essa del modello oralistico.

39. Quinn 1982; Habinek 2005, Wiseman 2015.

40. Parker 2009, 187 s. Da parte mia avevo sostenuto una posizione analoga in Citroni 1995, 9-12.

41. Miller 1994, 191 fa discendere dalla premessa secondo cui un carme che si sia conservato deve aver avuto ragioni di interesse che andavano al di là di quelle poste dal momento iniziale di enunciazione (vedi sopra, n. 36) la considerazione, che credo giusta ma che non credo derivi necessariamente da quella premessa, che "quando si parla del contesto perfomativo di un carme non si deve intendere un evento individuale concreto ma piuttosto dei tipi di situazione in cui un poema di quel tipo potrebbe essere stato convenientemente e logicamente eseguito".

\section{RIASSUNTI}

Heinze (nel saggio Die horazische Ode), Fraenkel (nell'Horace) e gli studiosi influenzati dal New Criticism concordavano nel rifiutare il ricorso a elementi contestuali esterni al testo nell'interpretazione delle odi di Orazio, ma questa posizione critica aveva, in ciascuno dei tre casi, origini e motivazioni sostanzialmente diverse che vengono qui analizzate. In Heinze, in Fraenkel, ma anche in fasi precedenti del dibattito, e poi in fasi successive fino ad oggi, nell'interpretazione della lirica antica e del suo rapporto con occasioni e destinatari entrano in gioco delle opposizioni, intese rigidamente: quella tra la lirica antica che avrebbe carattere dialogico e la lirica moderna che avrebbe carattere fonologico; quella tra la lirica greca che sarebbe caratterizzata dalla dimensione orale e perciò da un legame diretto con l'occasione e col destinatario e la lirica latina che sarebbe invece 'libresca' e in cui occasione e destinatario andrebbero perciò considerati solo in quanto elementi della finzione letteraria-formale del componimento. Queste opposizioni vengono qui messe in discussione, e largamente sfumate, anche nel confronto con posizioni espresse in studi recenti e recentissimi sulla lirica antica e moderna (specialmente di M. Lowrie, A. Barchiesi, P.A. Miller, P. Alpers, J. Culler). Si sostiene infine la rilevanza dell'occasione e del destinatario nell'interpretazione della lirica oraziana almeno nei casi in cui siano coinvolti personaggi reali viventi al tempo della composizione: personaggi e situazioni subiscono un processo di trasfigurazione e tipizzazione che li rende idonei a integrarsi in testi di natura essenzialmente estetica, destinati a un pubblico generico, ma che non li astrae del tutto dalla dimensione storica e biografica. La conoscenza, quando possibile, dei dati di realtà consentirebbe, a noi, e consentiva in modo differenziato a diverse componenti del pubblico antico, di comprendere meglio il processo, propriamente artistico, di trasfigurazione e tipizzazione. 
INDICE

Mots-clés : Lirica greca, Lirica latina, Oralità, Occasione, Destinatario, Richard Heinze, Eduard Fraenkel, Philip Buttmann, New Criticism, Pubblico, Performance / Reperformance 